\title{
A Case of Aripiprazole Induced Tardive Dyskinesia in a Neuroleptic-Naïve Patient with Two Years of Follow Up
}

\author{
Rakesh Goyal ${ }^{1}$, Salam Hemabati Devi ${ }^{2}$ \\ 'Institute of Living/Hartford Hospital, Hartford, CT, USA, ²Department of Psychiatry, Lady Hardinge Medical College and Hospitals, New \\ Delhi, India
}

\begin{abstract}
Tardive dyskinesia (TD) is arguably the most serious and potential irreversible side effect of antipsychotic medication. Traditionally first generation antipsychotics are the neuroleptics considered to have higher risk of TD as compared to second and third generation antipsychotics. Aripiprazole is a third-generation antipsychotic with a novel mechanism of action. Risk of developing TD with use of aripiprazole has been unknown. Recently many cases of aripiprazole associated TD have been reported. A case of 52 year old Caucasian woman is discussed who presented to us with first manic episode. Patient had never been treated with any antipsychotic medication in her life before. During current episode, she was treated with aripiprazole $30 \mathrm{mg} / \mathrm{day}$. During follow up, patient was found to have developed dyskinetic oro-facial movements within 2 months of starting aripiprazole. She was not taking any other antipsychotic/anti-dopaminergic medication at that time. Patient's abnormal oro-facial movements could not be reversed in spite of immediate discontinuation of aripiprazole. Multiple medications are tried over the next 2 years but her movement disorder never remitted. Above case (along with other recent reports) suggest that risk of movement disorder with aripiprazole use could be higher than previously thought. Further studies are required to find out incidence of movement disorder with aripiprazole. Aripiprazole use should be preferably restricted to FDA approved indications. Clinician needs to be very vigilant about emergence of any movement disorder while using aripiprazole, especially in patients with risk factors for TD.
\end{abstract}

KEY WORDS: Tardive dyskinesia; Aripiprazole; Antipsychotics side effects; Movement disorders.

\section{INTRODUCTION}

Tardive dyskinesia (TD) is arguably the most disabling and potentially irreversible movement disorder associated with antipsychotic medication use. Traditionally, first generation antipsychotics (FGA) are associated with risk of developing TD. Newer antipsychotics (second and third generation) were introduced with a promise of having much lower risk of TD but questions have been raised against this claim recently. ${ }^{1)}$

Aripiprazole is a third-generation antipsychotic with a novel mechanism of action involving mixture of agonist, partial agonist and antagonist activity at dopamine and serotonin receptors. ${ }^{2)}$ Aripiprazole is FDA approved for treatment of schizophrenia, bipolar I disorder and as an

\footnotetext{
Received: September 16, 2013/Revised: November 29, 2013

Accepted: December 2, 2013

Address for correspondence: Rakesh Goyal, MD

Braceland Bldg \#234, Institute of Living/Hartford Hospital, 200

Retreat Avenue, Hartford, CT 06106, USA

Tel: +1-860-545-7239

E-mail: rkg8910@gmail.com
}

adjunct for major depressive disorder. Being a recently introduced medication (in 2003), risk of movement disorders with aripiprazole use is unknown. ${ }^{1)}$ Till now, aripiprazole has been regarded as a relatively safe agent when it comes to the risk of developing TD. In fact few have reported aripiprazole to be therapeutic for TD. ${ }^{3,4)}$ However, by contrast, there is slowly increasing published data which associates aripiprazole with TD ${ }^{5-7)}$ Emergence of aripiprazole-induced TD in a neuroleptics naïve patient can be a stronger association in that regard. The following is a case of aripiprazole-induced early onset (within 2 months) TD in a middle aged woman with no past exposure to antipsychotics or history of any neurologic disease.

\section{CASE}

A 52 year-old woman presented to our hospital (University Hospital, Newrak, New Jersey) in February 2011 with complaints of irritable, anxious mood and inability to focus for one month. She also gave history of de-

(c) This is an Open-Access article distributed under the terms of the Creative Commons Attribution Non-Commercial License (http://creativecommons.org/licenses/by-nc/3.0) which permits unrestricted non-commercial use, distribution, and reproduction in any medium, provided the original work is properly cited. 
creased need for sleep and excessive spending in the same time period. On exam, she was well groomed, had fast speech but not pressured, irritable and labile affect but no grandiose ideas.

Patient denied ever having similar symptoms in her life before. Treatment history revealed that patient had taken nefazodone, bupropion, duloxetine and clonazepam but has never taken any antipsychotic medication in her life. Her routine laboratory examinations (complete blood count, liver function test, and urine analysis) were within normal limits. CT scan of head and brain (without contrast) was negative for any acute changes or other pathology.

Patient was diagnosed with bipolar disorder with current episode of mixed type. Patient did not want to take lithium or valproate medications fearing about their side effects. She was started on aripiprazole $15 \mathrm{mg}$ at bedtime which was increased to $15 \mathrm{mg}$ twice a day after 4 days. Patient started feeling better and was discharged on day 10 with aripiprazole $30 \mathrm{mg} /$ day at bedtime and clonazepam $0.5 \mathrm{mg}$ twice a day.

On patient's first follow up visit no medication side effects were reported or observed. On her second follow up visit, she complained of disturbing motor symptoms which included intermittent involuntary painful jaw clinching and abnormal tongue movements. Physical exam showed involuntary chewing movements of jaw, lip smacking, infrequent tongue protrusion and twisting and side to side movement of the tongue. Patient scored 14 on abnormal involuntary movement scale (AIMS). She had never experienced these symptoms before in her life and was not taking any other medications which could explain these extrapyramidal symptoms. Serum copper, serum ceruloplasmin and serum thyroid stimulating hormone levels were found to be in normal range.

Aripiprazole was stopped immediately but patient continued to have abnormal involuntary movements affecting face, tongue and lips. In next 2 years, patient was treated with various psychotropics including ziprasidone, quetiapine, lamotrigine and sodium valproate to control her mood symptoms which required admission to inpatient psychiatry units. Patient never had remission of her TD symptoms.

On last encounter, in March 2013, examination showed that now she additionally had repetitive non-rhythmic flexion-extension movements in her toes. She scored 20 on AIMS now. Patient was currently taking sodium valproate $1,500 \mathrm{mg} /$ day and quetiapine $200 \mathrm{mg} /$ day.

\section{DISCUSSION}

We made some important observations in the above described patient. First, above patient was antipsychotic medications naïve till she took aripiprazole. Second, she developed classical TD like abnormal involuntary movements within 2 months of starting therapy with aripiprazole. There was no other medication in her regimen which could explain her abnormal oro-mandibular movements. Third, TD symptoms did not remit after discontinuing aripiprazole.

According to most widely held theory, pathophysiology of TD involves increased dopamine receptor sensitivity (possibly associated with D2 receptor up-regulation) following chronic blockade of the receptors, particularly in the frontal-subcortical motor circuit. ${ }^{8)}$

One possible mechanism to explain risk of TD with aripiprazole may be its novel pharmacodynamic properties. Recent studies have proposed that the aripiprazole, instead of being only a partial agonist at D2 receptors, is 'functionally selective' at D2 receptors which means depending upon the cellular milieu a mixture of agonist/partial agonist/antagonist actions can occur. ${ }^{2}$ In this regard, it is important to note that researchers have also found aripiprazole to have much higher affinity for the human dopamine D2 receptor than other atypical antipsychotic drugs with aripiprazole occupying up to $90 \%$ of D2 receptors. ${ }^{9}$

The property of aripiprazole to act "functionally selectively" at D2 receptors with high affinity for D2 receptors may result in aripiprazole behaving like high potency FGAs in certain class of patients. Which patients are at risk of developing TD with aripiprazole is still a matter of research. Of the currently proposed risk factors for TD, our patient had few of them, i.e. female gender, relatively old age and having an affective disorder.

Regarding aripiprazole being useful in alleviating symptoms of TD related with other antipsychotic agents, ${ }^{3,4)}$ one possible mechanism for such observation may be suppression of TD in short term by high dopamine receptor binding activity of aripiprazole or spontaneous, coincidental remission of TD rather than a true therapeutic effect.

Controversy continues to exist as there are reports both for and against the risk of TD with aripiprazole use. Nearly all of the published data is in the form of case reports. In most of the reports patients were exposed to multiple neuroleptics, making the identification of culprit medication difficult. Patient described above was neuroleptic naïve. 
This report suggests that aripiprazole carries the risk of causing TD especially in patients with known risk factors for TD. Clinician should be aware of this risk and remain vigilant. Long term prospective follow up data from a large group of patients using aripiprazole can help clear the controversy.

\section{REFERENCES}

1. Tarsy D, Baldessarini RJ. Epidemiology of tardive dyskinesia: is risk declining with modern antipsychotics? Mov Disord 2006;21:589-598.

2. Shapiro DA, Renock S, Arrington E, Chiodo LA, Liu LX, Sibley DR, et al. Aripiprazole, a novel atypical antipsychotic drug with a unique and robust pharmacology. Neuropsychopharmacology 2003;28:1400-1411.

3. Kang NR, Kim MD. Tardive dyskinesia: treatment with aripiprazole. Clin Psychopharmacol Neurosci 2011;9:1-8.

4. Osorio RS, Agüera-Ortiz L, Hurtado de Mendoza A, Ramos I, Palomo T. Treatment of tardive dyskinesia with aripiprazole. Neurotox Res 2010;17:432-434.

5. Hall DA, Agarwal P, Griffith A, Segro V, Seeberger LC. Movement disorders associated with aripiprazole use: a case series. Int J Neurosci 2009;119:2274-2279.

6. Peña MS, Yaltho TC, Jankovic J. Tardive dyskinesia and other movement disorders secondary to aripiprazole. Mov Disord 2011;26:147-152.

7. Wang LJ, Ree SC, Chen CK. Courses of aripiprazoleassociated tardive dyskinesia: report of two cases. Prog Neuropsychopharmacol Biol Psychiatry 2009;33:743-744.

8. Klawans HL Jr, Rubovits R. An experimental model of tardive dyskinesia. J Neural Transm 1972;33:235-246.

9. Mace S, Taylor D. Aripiprazole: dose-response relationship in schizophrenia and schizoaffective disorder. CNS Drugs 2009;23:773-780. 